\title{
Reporte De Caso Clínico De Malformaciones Genéticas No \\ Especificadas En El Área De Neonatología Del Hospital General \\ Docente Ambato
}

\author{
Joselyn Monserrath Llanga Santana \\ joselynmonserrath26@gmail.com
}

Lic. Mg. Evelin Velasco

ef.velasco@uta.edu.ec

Universidad Técnica de Ambato

Ambato - Ecuador

\section{RESUMEN}

Objetivo: Identificar las malformaciones congénitas no especificadas mediante la revisión del historial clínico de una paciente del área de Neonatología del Hospital General Docente Ambato. La investigación se enmarcó en el tipo descriptivo, se recolectó información mediante la revisión de la historia clínica obtenida en el Hospital General Docente Ambato. Descripción del caso: se analiza a una recién nacida en el cantón Pelileo provincia de Tungurahua, quien a las dos horas posteriores al parto presentó dificultad respiratoria, siendo ingresada de emergencia al Hospital General Docente Ambato, donde tras ser valorada por el personal médico es diagnosticada con malformaciones congénitas no especificadas, permaneció en la casa de salud por 44 días; sin embargo, pese a su deteriorado estado de salud fue retirada de la misma debido a la falta de compromiso de los padres con el tratamiento indicado por el personal de salud. En conclusión: las anomalías congénitas son patologías provocadas por varios factores encontrados en el entorno de la madre, por ende, la insuficiente detección a tiempo de esta patología genera consecuencias inevitables para el recién nacido, como también causa un impacto psicológico negativo en sus progenitores.

Palabras clave: anomalías congénitas; patologías; recién nacida; síndrome; teratógeno. 


\title{
Clinical Case Report Of Unspecified Genetic Malformations In The Neonatology Área Of The Hospital General Docente Ambato
}

\begin{abstract}
Objective: To identify unspecified congenital malformations through the review of the clinical history of a patient in the Neonatology area of the Hospital General Docente Ambato. The research was framed in the descriptive type, information was collected through the review of the clinical history obtained at the Hospital General Docente Ambato. Case description: a newborn girl was analyzed in the Pelileo city, province of Tungurahua, who two hours after delivery presented respiratory difficulty and was admitted to the Hospital General Docente Ambato, where after being evaluated by the medical staff she was diagnosed with unspecified congenital malformations. She remained in the hospital for 44 days; however, despite her deteriorated state of health she was removed due to the lack of commitment of the parents with the treatment indicated by the health staff. In conclusion: congenital anomalies are pathologies caused by several factors found in the mother's environment, therefore, insufficient early detection of this pathology generates inevitable consequences for the newborn, as well as causing a negative psychological impact on the parents.
\end{abstract}

Key words: congenital anomalies; pathologies; newborn; syndrome; teratogen.

Artículo recibido: 02 noviembre. 2021 Aceptado para publicación: 28 noviembre 2021 Correspondencia: joselynmonserrath26@gmail.com Conflictos de Interés: Ninguna que declarar 


\section{INTRODUCCIÓN}

La gestación es un período de tiempo que inicia desde la concepción hasta el nacimiento, pues, es aquí donde se produce un acontecimiento llamado organogénesis, relacionado con desarrollo anatómico, estructural y morfológico del embrión, que comprende a partir de la cuarta a octava semana de embarazo, siendo indispensable que la madre tome los cuidados adecuados, considerando que el feto tiene grandes posibilidades de sufrir algún tipo de alteración (Serna, 2012).

Las malformaciones genéticas denominadas también defectos de nacimiento o trastornos congénitos, son anomalías funcionales, estructurales y metabólicas que ocurren durante la vida intrauterina y son detectadas durante el proceso de gestación o posteriores al parto (Rojas et al., 2012). Según un estudio realizado por la Organización Mundial de la Salud (OMS), se ha demostrado que cada año 303.000 recién nacido a nivel mundial fallecen debido a esta causa (OMS, 2020).

En países de Latinoamérica estos trastornos ocupan el décimo lugar como causa de muertes neonatales pudiendo tener origen genético, infeccioso o ambiental, en la mayoría de circunstancias resulta muy complejo identificar los factores que lo provocan, siendo posible prevenirlos implementando medidas fundamentales como la ingesta adecuada de alimentos, ácido fólico, calcio y yodo durante el primer trimestre de embarazo, la vacunación y en especial el control prenatal que es primordial durante el período gestacional. De igual manera es imprescindible que la madre no se exponga a sustancias dañinas como agentes químicos que pongan en riesgo su salud, así como la del feto (Carrallo \& Ares, 2018).

El nacimiento de un neonato que presenta anomalías congénitas provoca ansiedad, preocupación y en varias ocasiones rechazo hacia el mismo, al causar una disrupción del entorno familiar, pues para los padres, aceptar esta condición es uno de los puntos más difíciles de tratar. Al respecto, es recomendable que la persona encargada de brindar atención durante el parto o el médico informe a la familia sobre el estado del niño al momento del nacimiento, educándose en el ámbito psicológico para enfrentar la situación (Chaves et al., 2018).

Los defectos congénitos inducidos por sustancias químicas representan aproximadamente el 5\% de la mayoría de este tipo de patologías presentes en recién nacidos, pues antes de establecerlo como factor etiológico debe considerarse que el agente químico atraviese la 
placenta de manera que pueda influir completamente en la formación intrauterina del nuevo ser (Ortiz et al., 2015).

Existe una variedad de teratógenos que son agentes ambientales capaces de causar daños al embrión, entre ellos se mencionan las drogas ilícitas, fármacos, enfermedades propias de la progenitora o compuestos químicos, en especial las sustancias que se encuentran en grandes industrias textiles, donde laboran mujeres en estado de gestación sin los equipos de protección personal (EPP) adecuados para realizar este tipo de trabajos, considerando que requieren de técnicas especiales para ser manipulados, además, la sobreexposición tiene graves repercusiones en el recién nacido (Rodríguez et al., 2019).

En la provincia de Tungurahua - Ecuador se ubica el cantón Pelileo conocido como cuidad "Azul", sitio donde grandes fábricas confeccionan cantidad de prendas de vestir entre ellas sobresale el JEAN, que en la actualidad es utilizado por el consumidor, constituyéndose en una de las fuentes de mayor economía para la población que vive en el sector. Estas empresas utilizan sustancias químicas en su elaboración, mencionándose las siguientes: metalsulfito de sodio, peróxidos, cloro, ácido fórmico, sosa caustica, ácido oxálico y tinturas, que cumplen una función específica en el tinturado, considerando que hasta adquirir el color característico del Jean (azul) entre otros tonos, es necesario efectuar un sin número de lavados y prelavados denominados STON o SUPER STONES (lavado en piedra), proceso fisicoquímico de una prenda antes de su comercialización (López, 2012).

Es menester resaltar que, dentro del campo manufacturero existe déficit de conocimiento sobre la importancia que tiene el EPP, pues en varias de estas empresas no son utilizados de la manera adecuada, o en el peor de los casos no abastecen a sus empleados de este tipo de prendas. La seguridad industrial determina la responsabilidad del empleador en proporcionar equipos que ayuden a sus dependientes a realizar sus actividades, en especial cuando laboran mujeres embarazadas, por lo tanto es prioritario el respeto hacia sus derechos, debiendo brindar las condiciones y protecciones necesaria, con el propósito de evitar o disminuir la exposición a peligros físicos, químicos, eléctricos, radioactivos o accidentes mecánicos, elementos esenciales que garantizan la seguridad del trabajador en la industria (Moretta, 2017). 


\section{METODOLOGÍA}

La investigación se enmarcó en la modalidad bibliográfica documental, al realizarse una revisión de literatura en diferentes repositorios, revistas científicas y documentales, que aportaron en la fundamentación teórica, aspecto que benefició en la profundización del conocimiento relacionado con la temática propuesta. Además, fue de campo, porque la investigadora acudió al lugar donde sucedieron los acontecimientos como es el Hospital General Docente Ambato, de esta forma recolectó la información necesaria que dio realce al estudio propuesto.

El tipo de investigación aplicado fue el descriptivo, que benefició en la recolección de datos de la historia clínica obtenida del área de Neonatología del HOSPITAL GENERAL DOCENTE AMBATO ubicado en la provincia de Tungurahua, cantón Ambato, posibilitó la profundización en el caso clínico de una recién nacida que presentó anomalías congénitas.

\section{DESCRIPCIÓN DEL CASO}

Paciente nacida en el Hospital Básico de Pelileo provincia de Tungurahua-Ecuador a las 4:55 AM del 26 de mayo del 2021 producto de un parto eutócico, Apgar de 8 - 9, peso: $3215 \mathrm{gr}$, talla: $47 \mathrm{~cm}$, perímetro cefálico: $35 \mathrm{~cm}$, se colocó profilaxis ocular y hemorrágica, pasó a alojamiento conjunto con su madre y a las dos horas posteriores al parto presentó saturación del $60 \%$, siendo trasladada de emergencia al Hospital General Docente Ambato por presentar dificultad respiratoria, se administró oxígeno a 1 litro por cánula nasal llegando a saturar de 80 a $85 \%$ además se observó leves retracciones costales, decidiendo su ingreso al área de Neonatología.

Durante la entrevista realizada a los padres, estos refirieron no tener antecedentes patológicos, familiares o quirúrgicos, no consumo de drogas o cualquier otra sustancia psicotrópica, ocupación obreros y maquiladores en una fábrica de jeans por más de 3 años.

Con respecto a los antecedentes ginecoobstétricos la madre refirió tener 2 gestas: la primera hace 1 año y 6 meses y la segunda sería la actual, un embarazo no planificado, donde únicamente se realizó 2 controles prenatales más 2 ecografías, entre las vitaminas y minerales recibió hierro y ácido fólico, 1 sola dosis de vacuna antitetánica y no tuvo complicaciones durante el período de gestación. 
Examen físico: cabeza normocefálica, fontanela anterior normotensa, apertura ocular espontánea, cuello corto, cabello y orejas de baja implantación, paladar: alto y estrecho; Tórax: simétrico con buena expansibilidad, corazón rítmico no soplos.

Abdomen: suave no visceromegalias, no distendido, ruidos presentes, cordón umbilical con dos arterias y una vena.

Extremidades superiores: con tono y movilidad conservada manos en clinodactilia y camptodactilia.

Genitales: labios mayores cubren a los menores, pie equino varo derecho bilateral, reflejo de succión débil, búsqueda palmar como plantar presentes, además de buen sostén cefálico.

De acuerdo con la valoración realizada mediante el examen físico se observaron varias alteraciones, los diagnósticos médicos asignados fueron los siguientes: síndrome de dificultad respiratoria, problemas de alimentación (déficit de deglución), anomalía cromosómica no especificada y pie equino varo.

Se le realizaron varios estudios complementarios con los siguientes resultados: Eco transfontanelar: normal; Resonancia magnética: no lesiones hemorrágicas, isquémicas ni ocupativas, mastoiditis de forma bilateral; Ecocardiografía: normal; Eco abdominal: sin datos patológicos, Cariotipo: normal, no se descarta alteraciones cromosómicas de otro tipo. Este último examen evidenció la presencia de 46 cromosomas no afectados, sugiriéndose un asesoramiento genético.

A su ingreso, la paciente fue colocada en una cuna de calor radiante donde se encontraba hipoactiva, reactiva al manejo, flácida, mal sostén cefálico, débil reflejo se succión y deglución, con presencia de abundantes secreciones en vías respiratorias altas, dificultad respiratoria, saturando 60\%, cianosis, taquipnea, más retracciones costales marcadas, además de realizar picos febriles y taquicardias.

Se colocó oxígeno por cánula nasal con una $\mathrm{FiO} 2$ del $28 \%$ manteniendo parámetros normales de saturación, además fue colocada una sonda orogástrica por la cual se alimentó con leche materna, según criterio médico cada 3 horas con buena tolerancia oral, se realizó aspiración de secreciones bucales en cada manejo y por razones necesarias debido a la malformación que presentaba en el paladar lo cual impedía una correcta oxigenación. 
El deterioro clínico de la paciente se evidenciaba con el paso de los días, pues el problema para alimentarse ocasionó que existan variantes con respecto a su peso, la dificultad respiratoria se incrementó, siendo necesario colocar un CPAP nasal (presión positiva continua en las vías respiratorias) durante 10 días para mejorar la ventilación, sin embargo, no se obtuvo resultados positivos.

A los 17 días de vida presentó descompensación clínica con taquicardia, taquipnea y desaturaciones, se realizó exámenes de laboratorio los cuales reportan reactantes de fase aguda positivo: PCR: $24 \mathrm{mg} / \mathrm{dl}$, leucocitos $32.1600 \mathrm{~K} / \mathrm{ul}$, se recogió una muestra para urocultivo y se realizó una placa radiográfica de tórax, que confirmó una neumonía; por esta razón, se decidió colocar una terapia antibiótica de segunda línea con Linezolid y Meropenem.

El resultado del urocultivo reportó la presencia de Klebsiella Pneumoniae sensible a Gentamicina, decidiéndose continuar la terapia con antibióticos como: Linezolid y descontinuando el Meropenem.

Luego de superar el proceso infeccioso y permanecer en la sala de neonatología con variantes en su estado de salud, fue trasladada al servicio de pediatría para el manejo y cuidado materno; además, fue valorada nuevamente recibiendo como diagnóstico que la infante presentaba el síndrome de PIERRE ROBIN.

En el servicio de pediatría se mantuvo por el lapso de un día, pues los padres de la menor solicitaron en reiteradas ocasiones el alta voluntaria a pesar de conocer el estado clínico de la recién nacida. Esta medida fue analizada por el personal médico administrativo del Hospital General Docente Ambato junto con la sala de primera acogida, quienes notificaron el caso a la Junta Cantonal de la provincia de Tungurahua, entidad encargada de brindar protección integral a niños y adolescentes del Cantón Ambato, institución que resolvió se considere el alta voluntaria, según criterio del médico tratante, el cual accede a petición de los padres no sin antes dejar en claro el riesgo que tiene la niña al salir de la casa de salud, además de la responsabilidad de los progenitores por cualquier tipo de evento adverso que pueda suceder con respecto a la salud de su hija.

\section{DISCUSIÓN:}

Las anomalías congénitas no solo engloban a malformaciones estructurales, por el contrario también a defectos microscópicos, trastornos fisiológicos, errores metabólicos o anomalías moleculares y celulares. Este tipo de patologías afecta de manera importante 
el desarrollo del niño y constituye un tabú dentro de la sociedad, pues aún existe desconocimiento sobre el tema (Cajina, 2020).

Dentro de los factores socioeconómicos y demográficos se hace énfasis en la falta de recursos económicos de algunos países, pues se calcula que aproximadamente un $94 \%$ de anomalías congénitas graves se producen en mujeres que a menudo no poseen acceso suficiente a controles prenatales como también a alimentos nutritivos y son vulnerables a tener mayor riesgo a exposición a agentes o factores ambientales que aumentan la incidencia en el desarrollo prenatal anormal (Carrallo \& Ares, 2018).

La toxicidad en el desarrollo hace referencia fundamental a los efectos adversos que se producen durante el embarazo o que a su vez resulta de la sobreexposición que tienen los padres a sustancias dañinas que interfieren en la formación y producción de gametos sexuales de los mismos, en especial cuando han estado expuestos por un tiempo indefinido a compuestos químicos, en etapas de edad reproductiva (Petracchi, 2015).

Según la normativa NTP 542 (guías de buenas prácticas) consideran agentes peligrosos para la reproducción "a toda sustancia que pueda afectar a la salud reproductiva del hombre como de la mujer" influyendo en la capacidad de las parejas para tener niños sanos. Un compuesto químico tóxico para la reproducción es aquel que por ingestión, inhalación o penetración cutánea puede producir efectos negativos no hereditarios en la descendencia, afectando a la función o a la capacidad reproductora.

El síndrome de Pierre Robin es un tipo de anomalía congénita del arco branquial asociada a la glosoptosis (desplazamiento de la lengua hacia la parte posterior de la cavidad bucal), micrognatismo (mandíbula más pequeña de lo normal) y a la hendidura del paladar, los niños que lo padecen desarrollan desde el nacimiento problemas respiratorios asociados directamente a una mortalidad elevada. Por ser una malformación del primer arco branquial se relaciona con varios problemas oftalmológicos, además del cuadro sistémico que presentan pueden comprometer al desarrollo del niño (Pérez \& García, 2012).

El manejo efectivo para este tipo de problema es determinante, debido a la incoordinación de los mecanismos de deglución y succión que van acompañados de un alto gasto energético que tiene como objetivo mantener una correcta ventilación respiratoria, es decir que, comprometen al estado tanto nutricional como la calidad de vida del paciente (Suárez et al., 2010). 
Los niños que presentan este tipo de síndrome tienen dificultad para alimentarse, considerando que disminuye la capacidad de la ingesta oral, lo que ocasiona el retraso del crecimiento y desarrollo, si el síndrome no es tratado a tiempo puede tener consecuencias graves como agotamiento, falla cardíaca y finalmente la muerte.

El tratamiento para esta alteración genética incluye una intervención quirúrgica o una terapia conservadora, pudiendo ser colocándolos en una posición prono hasta que exista un correcto crecimiento de la mandíbula, de esta manera impide que la lengua caiga hacia delante lo que facilita una correcta oxigenación al dejar libre la vía respiratoria. Por lo contrario si este tratamiento no funciona, es necesario tomar alternativas mediante tratamientos como traqueotomía, glosopexia o la expansión mandibular, conocida como distracción ósea si el caso amerita (Raffi, 2012).

Este defecto de nacimiento tiene una prevalencia aproximada de 1 en 8500 nacidos vivos, entre niños y niñas, una de las causas es la herencia autosómica recesiva, es decir una afección genética que se presenta cuando el niño hereda la copia de un gen mutado de cada uno de sus progenitores. La mutación de un gen ocurre con el cambio de la secuencia del ADN durante la división celular por causas como la exposición a radiación, sustancias químicas o infección por virus (Suárez et al., 2010).

Finalmente, el fuerte impacto psicológico que produjo en los padres de la recién nacida fue el rechazo hacia la misma, pues en varias ocasiones se le notificó especialmente a la madre para que realice apego materno y acuda al hospital a visitar y a conocer sobre el estado de salud en el que se encontraba su hija, a pesar de todos los intentos, no se obtuvo una respuesta positiva, de igual manera pese a la intervención psicológica que fue brindada en el hospital para conocer la situación, insistían que la niña estaría mejor en la casa que bajo cuidados médicos.

La falta de atención por parte de los progenitores ocasionó aislamiento durante el proceso de diagnóstico o terapéutico, desencadenando sentimientos de miedo, ira y resentimiento, afectando notoriamente a la estructura familiar, siendo que, cualquier tipo de malformación en el recién nacido genera una cantidad de preguntas o dudas, incluyendo cuestionamientos acerca de la causa de la enfermedad, el origen genético familiar del padre como de la madre, las consecuencias de estas patologías en el desarrollo psicológico del individuo, además de la posible recurrencia de este tipo de anomalías en subsiguientes embarazos (Ballesteros et al., 2006). 


\section{CONLUSIÓN O CONSIDERACIONES FINALES}

La gestación es un período de tiempo importante para cada mujer, pues es aquí donde ocurre un sin número de acontecimiento en el organismo para dar vida a un nuevo ser; es decir, es una etapa de vulnerabilidad debido al constante cuidado con el entorno que la rodea; existen diferentes factores ambientales, sociales y psicológicos que interfieren en el desarrollo del embarazo que generan consecuencias perjudiciales para la salud tanto de la madre como de su hijo; cabe señalar que la exposición a sustancias tóxicas aumentan el riesgo significativamente en el desarrollo embrionario, pues agentes como drogas, medicamentos, compuestos químicos, exposiciones ocupacionales o del medio ambiente son el detonante principal de consecuencias graves como muertes neonatales y malformaciones genéticas en los infantes.

Las malformaciones genéticas son temas con mayor complejidad para tratarlos dentro de la sociedad, además, se caracteriza por constituirse en un problema de salud pública que afecta a la población mundial; en la actualidad existen varios casos reportados en recién nacidos que padecen esta patología, así la falta de información sobre el tema ha traído consecuencias negativas en innumerables familias pues ninguna ha estado preparada para recibir un diagnóstico patológico; es decir que varias madres no cuentan con la información suficiente sobre el estado de salud que presenta su hijo durante el periodo de embarazo o en varias ocasiones no cuentan con los recursos necesarios para realizarse un control prenatal adecuado cada trimestre que permita conocer sobre la salud en el que se encuentra su hijo, por lo cual al momento del nacimiento reciben noticias devastadoras con respecto a la situación actual del infante, provocando problemas dentro del entorno que la rodea, además de desencadenar trastornos psicológicos como ansiedad, depresión, dudas, miedo, rechazo o en varias situaciones el abandono del recién nacido.

El actuar del personal de salud es indispensable en este tipo de situaciones en especial por parte de enfermería, pues como profesión tienen la capacidad para promover la salud mediante la educación al paciente en diferentes temas que ayuden a mejorar el conocimiento del mismo, en este caso la enfermera cumple la función fundamental de fortalecer el vínculo entre la familia y el recién nacido que presenta este tipo de anomalías congénitas, implementado actitudes humanísticas, éticas y de responsabilidad que permitan mejorar la relación de los mismos y así garantizar bienestar tanto de su paciente como de los progenitores. 
Actualmente la educación para la salud incluye el área de enfermería que cumple un rol trascendental en la profesión, al contribuir en los problemas de cronicidad, con el seguimiento de los procesos, activación y la gestión de recursos asistenciales; en el aspecto humano y material es fundamental mencionar que su función formativa se encuentra basada en el autocuidado, en esta misma línea considerando que su perfil universitario se encuentra vinculado con la educación orienta al bienestar de las familias; dentro de la labor social se relaciona con las necesidades poblacionales de la salud, enfocando su aporte al sector vulnerable con planes y programas preventivos que favorece en el mejoramiento de la calidad de vida del paciente y la comunidad.

Agradecimientos: Agradezco al Señor Director del Hospital General Docente Ambato perteneciente al Ministerio de Salud Pública del Ecuador por permitir la realización de esta investigación, además mis sinceros agradecimientos a la Universidad Técnica de Ambato junto con sus docentes quienes han trasmitido sus conocimientos en especial a la Lic. Evelin Velasco por su aporte en la efectivización del presente estudio.

\section{LISTA DE REFERENCIAS BIBLIOGRÁFICAS}

Ballesteros, B., Gómez, M., Muñoz, L., Suárez, F., \& Castillo, E. (2006). PERSPECTIVA DEL CUIDADOR PRINCIPAL *. Univ. Psychol. Bogotá (Colombia), 5(3), 457473. http://www.scielo.org.co/pdf/rups/v5n3/v5n3a03.pdf

Cajina, J. (2020). Malformaciones Congénitas Representación en Nicaragua. 1-6. https://iris.paho.org/bitstream/handle/10665.2/54204/boletinmalformaciones_spa .pdf? sequence $=1 \&$ isAllowed $=\mathrm{y}$

Carrallo, A., \& Ares, A. (2018). Las malformaciones congénicas. Influencia de los factores socioambientale en las diferentes comunidades autónomas. 1-39. https://www.unav.edu/documents/4889803/17397978/67_Orvalle_Enfermedade s+congénitas.pdf

Chaves, F., Vera, L., Rodrigues, S., Almeida, P., \& Carvalho, L. (2018). Ansiedad de madres de recién nacidos con malformaciones congénitas en los perdiodos pre y postnata. . RLAE, 1-8. https://doi.org/10.1590/1518-8345.2482.3080

López, O. (2012). "DETERMINACIÓN DE MEDIDAS DE PRODUCCIÓN MÁS LIMPIA PARA LA OPTIMIZACION DEL USO DE ENERGIA TÉRMICA Y CONSUMO DE AGUA EN LA INDUSTRIA DE LAVADO DE JEANS CHELOS PELILEO PROVINCIA DE TUNGURAHUA [Universidad Técnica de Ambato ]. 
https://repositorio.uta.edu.ec/bitstream/123456789/1973/1/MSc.25.pdf

Moretta, A. (2017). "LOS AGENTES QUIMICOS Y SU INCIDENCIA EN LA SALUD DE LOS TRABAJADORES DEL PROCESO DE TINTURADO DE JEANS” [Universidad Técnicade Ambato]. https://repositorio.uta.edu.ec/bitstream/123456789/25982/1/Tesis_t1284mshi.pdf

OMS. (2020, December 1). Anomalías congénitas. https://www.who.int/es/newsroom/fact-sheets/detail/congenital-anomalies

Ortiz, F., Castro, E., Barraza, J., Manjarrez, G., Jímenez, G., \& Ruiz, F. V. (2015). Teratología y farmacoterapia durante el embarazo y lactancia. Med UAS, 6(1), 143. http://hospital.uas.edu.mx/revmeduas/pdf/v6/n1/teratologia.pdf

Pérez, J., \& García, Z. (2012). Panorama Cuba y Salud. 6(1), 44-46. http://www.redalyc.org/articulo.oa?id=477348944008

Petracchi, F. (2015). SOCIEDAD ARGENTINA DE DIAGNOSTICO PRENATAL Y TRATAMIENTO ACTUALIZACION SOBRE AGENTES TERATOGENOS. 1-18. http://sadipt.org/nuevaSadipt/html/docs/agenteTeratogenos.pdf

Raffi, D. (2012). Reporte de caso Pierre Robin . Odontol Pediatr, 11(2), 149-153. http://repebis.upch.edu.pe/articulos/op/v11n2/a7.pdf

Rodríguez, M., Martalucia, B., Fernández, T., Rivadeneira, F., \& Tamayo Fernández, M. (2019). AGENTES TERATOGENICOS Y TERATOGENICIDAD AUTORES: Medica Genetista Profesora Asociada Instituto de Genética Humana. 1-30. https://www.javeriana.edu.co/documents/5782625/5901279/10++Teratogeno.pdf/8d22feb3-096b-4ddf-b31c-94a9bba1357c

Rojas, M., Rojas, L. W., \& Walker, M. \&. (2012). Malformaciones congénitas: aspectos generales y genéticos. Int. J. Morphol, 30(4), 1256-1265.

Serna, N. (2012). Biología del desarrollo.

https://accessmedicina.mhmedical.com/content.aspx? bookid=1476\&sectionid=9 5223528

Suárez, E., López, D., \& Sanz, H. (2010). Síndrome de Pierre Robin. Case Report, 33(1), 38-43. http://www.scielo.org.bo/scielo.php?script=sci_arttext\&pid=S101229662010000100008 